\title{
Feature-based attention influences later temporal perception
}

\author{
FUMINORI ONO \\ Juntendo University, Tokyo, Japan \\ Kyoko Yamada And Kazumitsu Chujo \\ Hiroshima University, Hiroshima, Japan \\ AND \\ JUN-ICHIRO KAWAHARA \\ National Institute of Advanced Industrial Science and Technology, Tsukuba, Japan
}

\begin{abstract}
We investigated the influence of feature-based visual attention on later temporal perception. Although there is ample evidence that space-based attention modulates temporal perception, it is not known whether feature-based attention also serves this function. The present study combined a visual selection task with a temporal interval production task to determine whether feature-based attention interacted with temporal perception. The results indicated that temporal perception of visual stimuli depended on whether the same stimulus had been attended to or ignored in a previous visual selection task. The temporal production of previously ignored stimuli was longer than the temporal production of either previously attended to or novel stimuli. This is the first demonstration of the effect of feature-based attention on later temporal perception. We concluded that temporal perception is affected by previously ignored stimuli.
\end{abstract}

Several factors can influence the processing of the temporal characteristics of a visual stimulus. For example, previous studies have revealed that the perceived duration of briefly presented stimuli increases with their number (Mo, 1975), size (Mo \& Michalski, 1972; Thomas \& Cantor, 1975), complexity (Schiffman \& Bobko, 1974), or familiarity (Avant, Lyman, \& Antes, 1975). That is, the subjective duration increases when observers perceive the stimuli as more numerous, large, complex, and/or familiar. In addition to these changes in the physical attributes of stimuli, more recent studies have shown the effect of attention on the temporal perception of visual stimuli. For example, Mattes and Ulrich (1998) briefly presented a precue to direct visual spatial attention to a specific location, followed by a probe dot with varying cue validity. The task was to categorize the duration of the dot as short, medium, or long. Among other important findings, the result most relevant to the present study was that observers' judgments of duration increased with stimulus probability, suggesting that the perceived duration of a stimulus in an attended location was longer than that of one in an unattended location (see also Chen \& O’Neill, 2001; Enns, Brehaut, \& Shore, 1999).

Although previous studies have presented robust effects of spatial attention on later temporal perception, the effect of feature-based attention on later temporal perception has not been studied. Because previous studies suggest that attention can also be selectively allocated to stimuli that match a particular feature, without shifts in the attended location (Moore \& Egeth, 1998; Treue \& Martínez-Trujillo, 1999), and because space-based attention interacts with temporal perception, it is worth asking whether featurebased attention also interacts with temporal perception. If so, we do not know whether feature-based attention and space-based attention affect temporal perception in the same ways. The present study was designed to examine this question.

We used the visual selection task devised by Raymond, Fenske, and Tavassoli (2003), who demonstrated that selective attention to a single stimulus in a pair of response alternatives modulates the subsequent emotional responses to that stimulus. Specifically, in Raymond et al.'s study, participants selected a target from two briefly presented stimuli, and then provided their emotional response (i.e., cheery or not) to a single test stimulus that had been presented in the previous selection task. It is important to note that the stimuli in the selection task were presented side by side, and the test stimulus in the estimation task was presented in the center of the screen. Thus, any difference in attending to or ignoring the stimuli should be attributable to the stimuli themselves. Results indicated that the stimuli previously presented as nontargets were

F.Ono, fuminori@med.juntendo.ac.jp 
rated as less cheerful than those previously presented as targets, suggesting that feature-based selective attention could drive emotional responses. In the present study, we adopted this procedure to direct selective visual attention to particular stimuli.

One benefit of adopting Raymond et al.'s (2003) selection task to study temporal perception is that the task allows us to examine the effects on temporal perception of both attended-to and ignored stimuli. Specifically, the objective of the present study was to compare the temporal perceptions at the ignored and attended locations, a comparison not previously available because previous studies had compared only the perceived durations of attended and unattended locations. In our study, each trial began with a selection task to direct participants' feature-based visual attention to one of two stimuli and was followed by a temporal production task. In the production task, a single test stimulus was presented, and the participant pressed a key when she or he judged that the prespecified interval (e.g., 2,000 msec) had elapsed from the onset of the test stimulus. The test stimulus was always either one of the stimuli that had been presented in the immediately preceding selection trial or a novel stimulus.

If feature-based attention affects later temporal perception functionally in the same way as space-based attention does, the perceived duration of previously attended stimuli, we predicted, would be prolonged. This prediction was based on studies suggesting that both types of attention behave similarly. For example, it has been shown that responses are faster and more accurate when feature-based attention is directed to a target (Kumada, 2001), or when the target is presented at locations to which space-based attention is directed (Downing, 1988; Müller \& Findlay, 1988). We hypothesized, therefore, that the duration of attended stimuli would be perceived as longer than that of unattended stimuli. On the other hand, if feature-based attention produces different effects on temporal perception, we predicted that the perceived duration of previously attended stimuli would not be prolonged, but would be less than, or the same as, that of the unattended stimuli.

\section{EXPERIMENT $1^{1}$}

\section{Method}

Participants. Fourteen experimentally naive students (age range, 18-28 years) from Hiroshima University volunteered in return for course credit or for pay. All had normal or corrected-to-normal visual acuity and had given their informed consent prior to participation. The participants were tested individually.

Apparatus and Stimuli. The stimuli were displayed on a CRT monitor (GDM-19PS, Sony, Tokyo) controlled by a PC/ATcompatible computer equipped with a frame store (VSG 2/5, Cambridge Research Systems, Kent, U.K.). The viewing distance was approximately $70 \mathrm{~cm}$.

Examples of the stimuli are shown in Figure 1. Stimulus conditions were the same as those used by Raymond et al. (2003). The novel complex visual stimuli were generated in every trial by randomly varying the elements that defined each as being one of four pattern types. Two of the pattern types were used as test images: "squares," created by randomly (with replacement) selecting the color of each section of a $5 \times 5$ grid from a 10 -color palette; and "circles," created by randomly varying the $x$ and $y$ coordinates and

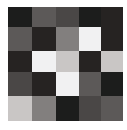

Squares

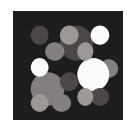

Circles

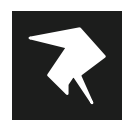

Polygon

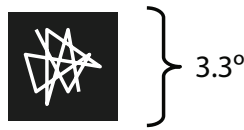

Squiggles
Figure 1. Examples of the stimuli used in the experimental trial and baseline trial.

radii of 20 uniquely colored circles on a gray background. The other pattern types were used only in blocks of baseline trials: "polygons," created by randomly varying the $x$ and $y$ coordinates of 10 vertices of the angles, forming an irregular red polygon on a gray background; and "squiggles," created by randomly varying the $x$ and $y$ coordinates of 10 points connected by a single red line on a gray background. Each image subtended approximately $3.3^{\circ} \times 3.3^{\circ}$ of visual angle. Single stimuli were presented at the center of the display. Pairs of stimuli were centered approximately $4.3^{\circ}$ of visual angle to the left and right of the display center.

Procedure. At the beginning of the experiment, each participant was asked to remove his or her watch and was told not to count during the temporal production task. After instructions were given, participants performed two types of practice trials for the purpose of studying the temporal interval $(2,000 \mathrm{msec})$ and learning the temporal production task. First, to familiarize participants with the interval of 2,000 msec, participants pressed a button to initiate a 2,000-msec presentation of a white square subtending approximately $3.3^{\circ} \times 3.3^{\circ}$ of visual angle. Participants repeated this procedure three times; following the third exposure of this first stage of practice, the participant initiated the second practice set by pressing the space bar. After a brief pause of $1,000 \mathrm{msec}$, the same white square appeared on the screen and remained until the participant responded. Participants were encouraged to estimate the same duration $(2,000 \mathrm{msec}$ from the onset of the white square) they had experienced in the first stage of practice as accurately as possible, and then to press the space bar again, which erased the display. A feedback display (too long, too short, or correct) appeared on the screen to encourage participants to respond more accurately. Correct was displayed when the response fell within a $10 \%$ temporal window centered in the 2,000 -msec interval of 1,900 to $2,100 \mathrm{msec}$.

After 20 trials of second-stage practice, participants performed the first trial. A trial consisted of the selection and the temporal production tasks. Participants pressed the space bar to begin a trial. Following presentation of a central fixation cross $(1,000 \mathrm{msec})$, the selection task display was presented for $100 \mathrm{msec}$, and then replaced by a blank screen. The display for the selection task consisted of a pair of stimuli positioned to the left and right of the fixation cross. In the no-exposure baseline trials the stimulus was a polygon pattern paired with a squiggles pattern, and in the experimental trials it was a pattern of squares paired with a pattern of circles. The positions (either left or right) of these stimuli were randomized across trials. At the beginning of each block, participants were informed of the target category (either polygon or squiggles for the baseline trials and either squares or circles for the experimental trials). In the selection task, the participants located the target pattern as quickly as possible by pressing the left or right arrow keys. (Note that in the baseline trials the target patterns on the temporal production task had never been presented during the selection task.) After a selection response, the participants pressed the space bar to begin a temporal production trial. Following the central fixation $(1,000 \mathrm{msec})$ presentation, a single test stimulus appeared in the center of the screen. In one third of the trials, the stimulus was the same image that had appeared as the target; in another third of the trials, the stimulus was the same image that had previously appeared as the distractor; in the final third of the trials, the stimulus was a novel image unlike either seen in the immediately preceding selection task. The display remained until the end of the temporal production task, which was terminated upon the keypress indicating that the temporal interval 
$(2,000 \mathrm{msec})$ had ended. It should be noted that the stimuli in the selection task were presented side by side and that the test stimulus in the temporal production task was presented in the center of the screen. Thus, any difference in attending to or ignoring the stimuli in the selection task would be attributable not to spatial attention but to feature-based attention. A sequence of events for this experimental trial is shown in Figure 2.

In the baseline trials, the polygon-and-squiggles patterns were presented in the selection task and the circles or squares were presented in the temporal production task; that is, in the temporal production task of the baseline trials, the participants saw a novel stimulus previously neither attended to nor ignored. These baseline trials enabled us to determine whether any differences in temporal perception represented a lengthening in the produced duration for stimuli previously ignored or a shortening in the produced duration for stimuli previously attended to.

Each participant was tested with two experimental blocks (60 trials per block) and two baseline trial blocks (30 trials per block). Block order and target category were counterbalanced. Before they started the second block, participants again performed 20 practice trials of the temporal production task.

\section{Results and Discussion}

We analyzed only production data for trials in which the targets had been correctly selected. The accuracy for the selection task was $99.2 \%$. Table 1 shows the mean produced interval for each previous attention state (ignored, attended, or baseline). A one-way ANOVA of attentionstate revealed a significant main effect $[F(2,26)=6.11$, $p<.01]$. The mean temporal production for the previously ignored stimulus was longer than that for the previously attended stimulus $[t(13)=2.87, p<.05]$ and for the novel stimulus $[t(13)=3.22, p<.01]$. The latter comparison is important because it indicates that previously ignoring a stimulus resulted in longer temporal production related to the novel stimulus. The difference between the mean temporal production for the previously attended stimulus and for the baseline was not significant $[t(13)=$ $0.822, p=.42]$. Figure 3 shows the mean produced intervals for attended trials minus baseline trials and that for ignored trials minus baseline trials. In terms of temporal experience, temporal production bears an inverse relationship to perceived duration; thus, overestimating temporal

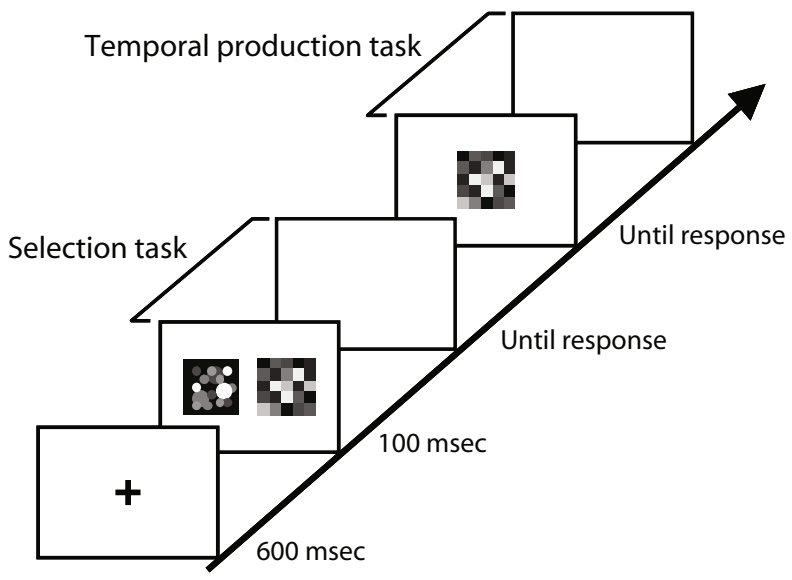

Figure 2. The sequence of events in an experimental trial.
Table 1

Mean Produced Interval (in Milliseconds) and Standard Errors for Each Previous Attention State in the Temporal Production Task of Experiment 1 Previous Attention State

\begin{tabular}{cccccccc}
\hline \multicolumn{2}{c}{ Attended } & & & \multicolumn{2}{c}{ Ignored } & & \multicolumn{2}{c}{ Baseline } \\
\cline { 7 - 8 }$M$ & $S E$ & & $M$ & $S E$ & & $M$ & $S E$ \\
\hline 2,642 & 165 & & 2,700 & 170 & & 2,657 & 172 \\
\hline
\end{tabular}

production is subjectively equivalent to underestimating perceived duration, and vice versa (Brown, 1997; Zakay, 1993).

It is worth noting that the temporal production for every previous attention state was longer than that for the trained duration. This pattern of results is consistent with previous literature, in that the temporal productions tend to become progressively longer across trials, a phenomenon called the lengthening effect (Hicks \& Allen, 1979; Ross, 1969).

The present finding - that temporal production for the previously ignored stimulus was longer than that for the previously attended stimulus and for the novel stimulusindicates that the perceived duration of the previously ignored stimulus was shorter than that of the previously attended stimulus and of the novel stimulus. These results suggest that feature-based attention affects temporal perception. Specifically, ignoring a visual stimulus decreased the perceived duration of the same stimulus when it appeared again.

\section{EXPERIMENT 2}

In Experiment 1, we found that previously ignoring visual stimuli shortened their perceived duration. An alternative explanation is that the duration for perceiving the ignored stimulus may have been delayed. Negative priming studies have shown that perceptual response is slower to previously ignored stimuli than to novel stimuli (Tipper \& Cranston, 1985). Psychophysiological studies have shown that stimuli associated with a no-go response cause a delayed response (e.g., Smid, Mulder, Mulder, \& Brands, 1992). The presentation of the ignored pattern associated with a no-go response might have caused a hesitant response, which then delayed the temporal production task. If the selection task in Experiment 1 led to negative priming or to hesitant response, it is conceivable that the longer temporal production duration for an ignored stimulus was due to a delay in perceiving the stimulus. To test this possibility, we used a perceptual discrimination task to evaluate perception speed. If the longer temporal production duration in Experiment 1 had been due to negative priming, the discrimination time for previously ignored stimuli should have been slower than for the baseline trial.

\section{Method}

Participants. Fourteen experimentally naive students (age range, 20-27 years) from Hiroshima University volunteered in return for course credit or pay. All had normal or corrected-to-normal visual acuity and had given their informed consent prior to participation. 


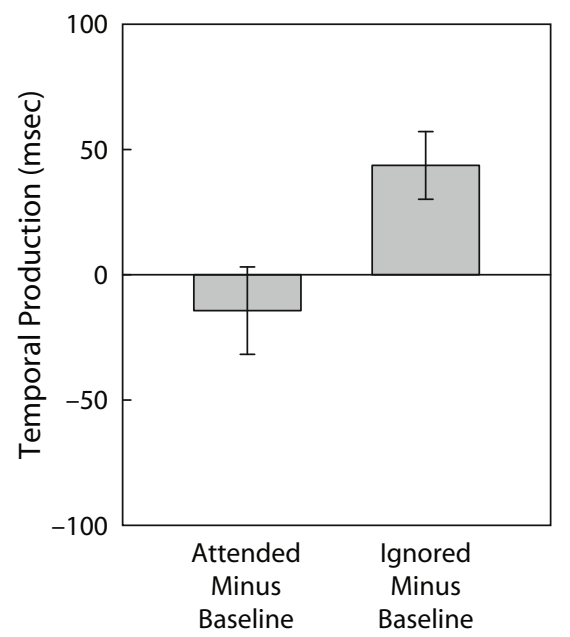

Figure 3. The mean produced intervals for attended trials and ignored trials, minus baseline trials, in the temporal production task of Experiment 1.

Procedure. All aspects of this experiment were identical to those in Experiment 1, except that a discrimination task was substituted for the temporal production task. Following a selection task, participants pressed the space bar to begin a discrimination trial. After the fixation display $(1,000 \mathrm{msec})$, a single test stimulus, either the circles or squares, appeared. A participant discriminated between squares or circles as quickly as possible by pressing a designated key. When the key was pressed, the discrimination display was erased. Each participant was tested with two experimental blocks (60 trials per block) and two baseline trial blocks (30 trials per block). Block orders and target categories were counterbalanced.

\section{Results and Discussion}

Error trials in the selection and the discrimination tasks were excluded from analyses. Accuracy for the selection and discrimination tasks was $98.5 \%$ and $97.6 \%$, respectively. Table 2 shows the mean reaction times (RTs) for each previous attention state - ignored, attended, or baseline - in the discrimination task. The main effect of the attention state was not significant $[F(2,26)=2.09$, $p=.14]$. The mean RTs for the previously attended stimulus were shorter, however, than for the baseline trials $[t(13)=2.17, p<.05]$. This result indicates that there was a conventional positive priming effect in which perceptual responses to previously attended stimuli were faster than perceptual responses to novel stimuli (Neely, 1977). The difference in RTs for the previously attended and previously ignored stimuli was not significant $[t(13)=1.2$, $p=.23]$. Finally, the difference in the RTs for the previously ignored and baseline trial stimuli was not significant $[t(13)=0.25, p=.80]$. There was no evidence that previously ignoring a stimulus produced a negative priming effect in the present selection task. This result also eliminated the possibility that the presentation of the previously ignored pattern caused hesitant response in the present study. Therefore, we concluded that the longer temporal production duration of ignored stimuli in Experiment 1 was due to the shortened perceived duration rather than to negative priming.

\section{GENERAL DISCUSSION}

The present study investigated the effect of feature-based visual attention on the later temporal perception of briefly presented stimuli. The results revealed that the selective ignoring of stimuli, as opposed to attending to them, influenced perceived duration. In particular, the perceived duration of previously ignored stimuli was shorter than for both novel stimuli and stimuli previously attended to. These results cannot be explained by negative priming, because RTs for the previously ignored stimuli were not different from those of the baseline trials in the discrimination task. Therefore, we suggest that feature-based attention affects later temporal perception differently from space-based attention. Specifically, ignoring stimuli in a feature-based manner shortened their perceived duration, and attending to stimuli in a feature-based manner produced no effect on temporal production. These effects - of feature-based ignoring and attending to stimuli-contrast sharply with those of space-based attending, which lengthened the perceived duration (the effect of space-based ignoring on temporal perception has not been examined yet).

It must be noted that in previous studies, space-based attention has been manipulated by precuing, and its lengthening effect has been revealed by comparing temporal perception performance at the cued and uncued locations (Chen \& O'Neill, 2001; Enns et al., 1999; Mattes \& Ulrich, 1998). Further studies are needed to examine whether temporal perception at an ignored location is shortened in the context of space-based attention as well.

Importantly, the present finding demonstrates that $s e$ lective attention, especially feature-based attention, shortens temporal perception. Regarding the two major conceptualizations of attention (Johnston \& Dark, 1986), most previous studies on temporal perception have focused on divided attention (for a review, see Brown, 1997). Typically, divided attention is created by a dual task in which participants' attentional resources are devoted to a primary task and the resources available for a secondary task (i.e., temporal perception task) are deprived. For example, participants in divided attention studies are often required to perform a primary task such as reading syllables, while simultaneously monitoring the duration of the primary task display. As a control condition, participants simply make a temporal judgment. In such circumstances, temporal estimates under dual-task conditions are generally shorter than those in the single-task condition (Macar, Grondin, \& Casini, 1994; Predebon, 1996; Underwood \& Swain, 1973; Zakay, 1993).

Table 2

Mean Reaction Times (in Milliseconds) and Standard Errors for Each Previous Attention State in the Discrimination Task of Experiment 2

Previous Attention State

\begin{tabular}{|c|c|c|c|c|c|}
\hline \multicolumn{6}{|c|}{ Previous Attention State } \\
\hline \multicolumn{2}{|c|}{ Attended } & \multicolumn{2}{|c|}{ Ignored } & \multicolumn{2}{|c|}{ Baseline } \\
\hline$M$ & $\overline{S E}$ & $M$ & $\overline{S E}$ & $M$ & $S E$ \\
\hline 578 & 36 & 591 & 37 & 593 & 38 \\
\hline
\end{tabular}


The distinction between selective and divided attention is important because models derived from studies that have examined the effect of divided attention on temporal perception - that is, the attentional allocation model (e.g., Brown, 1985; Hicks, Miller, \& Kinsbourne, 1976) cannot explain the present results. The attentional allocation model posits that temporal perception is influenced by the sharing of attentional resources between temporal and nontemporal processing. In this view, the more attention is devoted to temporal processing, the longer the perceived duration will be. In the present study, however, the stimulus for the temporal production task was presented by itself, with no concurrent task; therefore, attentional resources should have been fully devoted to temporal processing during the temporal production task, and production should have been the same across the attended, ignored, and baseline conditions.

A recent study provides evidence that, in the absence of a concurrent task, attention plays a role in the perception of duration. Tse, Intriligator, Rivest, and Cavanagh (2004) presented a stream of standard stimuli of equal duration. A stimulus differing in motion, color, or size was randomly inserted into this stream. The results showed that the estimated duration of items that were different and less frequent, therefore perceived as odd, was longer than the estimated duration of the standard stimuli, suggesting that the engagement of attention by an unexpected event might increase perceived duration. In the present study, however, the stimulus frequency was the same across the attended, ignored, and baseline trials; therefore, expectancy should have been the same across all three.

Although a full understanding of the mechanisms that underlie a decrease in perceived duration due to ignoring a previous stimulus must await further research, we can provide a tentative explanation for the present results. This explanation builds on a model of time perception proposed by Thomas and Weaver (1975), who claimed that perceived duration is a function of the amount of information processed during the objective time (see also Creelman, 1962; Treisman, 1963). According to these studies, there is an internal counter that keeps track of the number of units of temporal information processed for a given duration. This model assumes that when attention is deprived from monitoring the internal counter by engaging in other tasks, some proportion of the units of temporal information will probably be missed during the duration judgment. On the other hand, when attention is allocated to the monitoring, the duration judgment itself results in less temporal information being missed, thereby lengthening the apparent duration. We can interpret the present findings by integrating this idea and the effect of selective ignoring. Because selective ignoring is assumed to decrease representational salience of the previously ignored stimuli (Goldstein \& Fink, 1981; Rock \& Gutman, 1981), to access the ignored representation more attentional resources would be required, at the expense of resources needed for monitoring the internal counter. Such a shortage of attention for monitoring the counter results in the loss of the temporal information being tracked and a decrease in the perceived duration of previously ignored stimuli. In the present study, however, the previously attended stimuli were not perceived as lasting longer than were the baseline novel stimuli. Perhaps this was because the effect of attending to one of two stimuli was not intensive enough to influence the performance of monitoring the temporal counter under the current experimental procedure. Such an asymmetrical pattern of results for attending and ignoring could be a general effect when the present selection-task procedure is used. For example, Raymond et al. (2003), who used the same procedure as the present study, found a similar effect, in that affective judgment to previously attended stimuli was the same as that of baseline novel stimuli- - though the previously ignored stimuli were rated as less cheerful than baseline novel stimuli.

To summarize, we report two notable findings. The present results are the first to show the effect of featurebased visual attention on processing the temporal characteristics of a stimulus. This indicates that factors relating to feature-based attention should be included among the determinants affecting time perception of a stimulus display. Models of temporal perception require modifications to accommodate for the contribution of featurebased attention.

The second significant result of the present study is that selective ignoring, as opposed to selective attending, influences perceived duration. Further research is needed to investigate whether a similar effect would be obtained with space-based attention.

\section{AUTHOR NOTE}

This research was supported by a grant to F.O. from the Research Fellowships of the Japan Society for the Promotion of Science for Young Scientists. Correspondence concerning this article should be addressed to F. Ono, Department of Neurophysiology, School of Medicine, Juntendo University, 2-1-1 Hongo, Bunkyo-ku, Tokyo 113-8421, Japan (e-mail: fuminori@med.juntendo.ac.jp).

\section{REFERENCES}

Avant, L. L., Lyman, P. J., \& Antes, J. R. (1975). Effects of stimulus familiarity upon judged visual duration. Perception \& Psychophysics, 17, 253-262.

Brown, S. W. (1985). Time perception and attention: The effects of prospective versus retrospective paradigms and task demands on perceived duration. Perception \& Psychophysics, 38, 115-124.

Brown, S. W. (1997). Attentional resources in timing: Interference effects in concurrent temporal and nontemporal working memory tasks. Perception \& Psychophysics, 59, 1118-1140.

Chen, Z., \& O'Neill, P. (2001). Processing demand modulates the effects of spatial attention on the judged duration of a brief stimulus. Perception \& Psychophysics, 63, 1229-1238.

Creelman, C. D. (1962). Human discrimination of auditory duration. Journal of the Acoustical Society of America, 34, 582-593.

Downing, C. J. (1988). Expectancy and visual-spatial attention: Effects on perceptual quality. Journal of Experimental Psychology: Human Perception \& Performance, 14, 188-202.

Enns, J. T., Brehaut, J. C., \& Shore, D. I. (1999). The duration of a brief event in the mind's eye. Journal of General Psychology, 126, 355-372.

Goldstein, E. B., \& Fink, S. I. (1981). Selective attention in vision: Recognition memory for superimposed line drawings. Journal of Experimental Psychology: Human Perception \& Performance, 7, 954-967.

Hicks, R. E., \& Allen, D. A. (1979). Counting eliminates the repetition effect in judgments of temporal duration. Acta Psychologica, 43, 361-366. 
Hicks, R. E., Miller, G. W., \& Kinsbourne, M. (1976). Prospective and retrospective judgments of time as a function of amount of information processed. American Journal of Psychology, 89, 719-730.

Johnston, W. A., \& DARK, V. J. (1986). Selective attention. Annual Review of Psychology, 37, 43-75.

Kumada, T. (2001). Feature-based control of attention: Evidence for two forms of dimension weighting. Perception \& Psychophysics, 63, 698-708.

Macar, F., Grondin, S., \& CASIni, L. (1994). Controlled attention sharing influences time estimation. Memory \& Cognition, 22, 673-686.

Mattes, S., \& Ulrich, R. (1998). Directed attention prolongs the perceived duration of a brief stimulus. Perception \& Psychophysics, 60, 1305-1317.

Mo, S. S. (1975). Temporal reproduction of duration as a function of numerosity. Bulletin of the Psychonomic Society, 5, 165-167.

Mo, S. S., \& Michalski, V. A. (1972). Judgment of temporal duration of area as a function of stimulus configuration. Psychonomic Science, 27, 97-98.

Moore, C. M., \& Egeth, H. (1998). How does feature-based attention affect visual processing? Journal of Experimental Psychology: Human Perception \& Performance, 24, 1296-1310.

Müller, H. J., \& Findlay, J. M. (1988). The effect of visual attention on peripheral discrimination thresholds in single and multiple element displays. Acta Psychologica, 69, 129-155.

Neely, J. H. (1977). Semantic priming and retrieval from lexical memory: Roles of inhibitionless spreading activation and limitedcapacity attention. Journal of Experimental Psychology: General, 106, 226-254

Predebon, J. (1996). The relationship between the number of presented stimuli and prospective duration estimates: The effect of concurrent task activity. Psychonomic Bulletin \& Review, 3, 376-379.

Raymond, J. E., Fenske, M. J., \& Tavassoli, N. T. (2003). Selective attention determines emotional responses to novel visual stimuli. Psychological Science, 14, 537-542.

Rock, I., \& Gutman, D. (1981). The effect of inattention on form perception. Journal of Experimental Psychology: Human Perception \& Performance, 7, 275-285.

Ross, D. A. (1969). Lengthening of time estimates in four different patterns of visual light stimulation. Psychonomic Science, 16, 194-195.

SCHIFFMAN, H. R., \& BoвKo, D. J. (1974). Effects of stimulus complexity on the perception of brief temporal intervals. Journal of Experimental Psychology, 103, 156-159.
Smid, H. G., Mulder, G., Mulder, L. J., \& Brands, G. J. (1992). A psychophysiological study of the use of partial information in stimulus-response translation. Journal of Experimental Psychology: Human Perception \& Performance, 18, 1101-1119.

Thomas, E. A. C., \& Cantor, N. E. (1975). On the duality of simultaneous time and size perception. Perception \& Psychophysics, 18, 44-48.

Thomas, E. A. C., \& Weaver, W. B. (1975). Cognitive processing and time perception. Perception \& Psychophysics, 17, 363-367.

Tipper, S. P., \& CRanston, M. (1985). Selective attention and priming: Inhibitory and facilitatory effects of ignored primes. Quarterly Journal of Experimental Psychology, 37A, 591-611.

Treisman, M. (1963). Temporal discrimination and the indifference interval. Implications for a model of the "internal clock." Psychological Monographs, 77, 1-31.

Treue, S., \& Martínez-Trujillo, J. C. (1999). Feature-based attention influences motion processing gain in macaque visual cortex. Nature, 399, 575-579.

Tse, P. U., Intriligator, J., Rivest, J., \& Cavanagh, P. (2004). Attention and the subjective expansion of time. Perception \& Psychophysics, 66, 1171-1189.

Underwood, G., \& SwaIn, R. A. (1973). Selectivity of attention and the perception of duration. Perception, 2, 101-105.

ZAKAY, D. (1993). Time estimation methods: Do they influence prospective duration estimates? Perception, 22, 91-101.

\section{NOTE}

1. We conducted a pilot experiment to compare the temporal production for the previously attended stimuli and that for the ignored stimuli. Sixteen students participated in the experiment with the same procedure as used in Experiment 1, except that the baseline stimuli were not included. The results indicated that the mean temporal production for the previously ignored stimulus (mean, $2,598 \mathrm{msec}$; standard error, 134) was significantly longer than that for the previously attended stimulus (mean, $2,541 \mathrm{msec}$; standard error, 127) $[t(15)=2.83, p<.05]$. This pattern of results was consistent with the findings in the present study.

(Manuscript received January 10, 2006; revision accepted for publication September 1, 2006.) 